\title{
ON THE COEFFICIENTS IN ASYMPTOTIC FACTORIAL EXPANSIONS ${ }^{1}$
}

\author{
T. D. RINEY
}

1. Introduction. We shall consider the function

$$
{ }_{p} g_{q}(w)=\prod_{i=1}^{p} \Gamma\left(w+\sigma_{i}\right) / \prod_{j=0}^{q} \Gamma\left(w+\rho_{j}\right) .
$$

Here $p$ and $q$ are non-negative integers with $p \leqq q$; the $\sigma_{i}$ and $\rho_{j}$ denote arbitrary complex parameters and $w$ denotes a complex variable. It is known from a lemma which appears in the work of W. B. Ford [1], E. M. Wright [5], and H. K. Hughes [2] that ${ }_{p} g_{q}(w)$ admits an asymptotic factorial expansion in every right half-plane. Moreover, it follows from their investigations that the coefficients occurring in this expansion of ${ }_{p} g_{q}(w)$ are precisely the constants which occur in the asymptotic development for large $|z|$ of the important class of entire functions

$$
{ }_{p} G_{q}(z)=\sum_{n=0}^{\infty}{ }_{p} g_{q}(n) z^{n}
$$

However, the literature reveals no satisfactory method of determining these constants.

Van Engen [4] has utilized the fact that ${ }_{p} g_{q}(w)$ satisfies a first order difference equation in order to obtain a method of computing the coefficients. However, his procedure is greatly complicated by the introduction of intermediate constants which we shall avoid. By so doing, we obtain an explicit recursion formula for the coefficients in question.

2. Expansion of ${ }_{p} g_{q}(w)$. The expansion theorem due to the authors cited in $\$ 1$ is now stated without the proof which is based on Stirling's formula.

THEOREM 2.1. Let $h$ be any real number and let $M$ be any non-negative integer. Then in the half-plane $\operatorname{Re}(w)>-h$ with $|w|$ large, ${ }_{p} g_{q}(w)$ admits the asymptotic development

Received by the editors March 30, 1955 and, in revised form, May 3, 1955.

${ }^{1}$ Included in a thesis submitted to the Graduate School of Purdue University in partial fulfillment of the requirements for the degree of Doctor of Philosophy. 


$$
\begin{aligned}
{ }_{p} g_{q}(w)= & \left((2 \pi)^{1 / 2}\right)^{1-\alpha} \alpha^{\alpha w+\beta-1 / 2}\left[\sum_{m=0}^{M} \frac{c_{m}}{\Gamma(\alpha w+\beta+m)}\right. \\
& \left.+O\left(\frac{1}{\Gamma(\alpha w+\beta+M+1)}\right)\right]
\end{aligned}
$$

where $c_{0}=1$ and

$$
\alpha=q+1-p ; \quad \beta=\sum_{j=0}^{q} \rho_{i}-\sum_{i=1}^{p} \sigma_{i}+1 / 2(1-\alpha) .
$$

3. Two lemmas. We shall need the following two results:

Lemma 3.1. Let $\alpha$ be a positive real number and let $a$ and $b$ denote arbitrary complex parameters. Then the function $(w+a)^{-1} / \Gamma(\alpha w+b)$ has the convergent factorial expansion

$$
\frac{1}{(w+a) \Gamma(\alpha w+b)}=\frac{\alpha}{\Gamma(b-\alpha a)} \sum_{n=0}^{\infty} \frac{\Gamma(b-\alpha a+n)}{\Gamma(\alpha w+b+n+1)}
$$

in the right half-plane defined by $\operatorname{Re}(w+a)>0$.

PRoof. A proof of this well-known result is given in [1].

It will be convenient to introduce the notation

$$
R(w)=\prod_{i=1}^{p}\left(w+\sigma_{i}\right) / \prod_{j=0}^{q}\left(w+\rho_{j}\right) \quad(p \leqq q) .
$$

Then if $R(w)$ has only first order poles its partial fraction expansion is given by

$$
R(w)=\sum_{j=0}^{q} D_{j}\left(w+\rho_{j}\right)^{-1},
$$

where

$$
D_{i}=\prod_{i=1}^{p}\left(\sigma_{i}-\rho_{j}\right) / \sum_{k=0, k \neq j}^{q}\left(\rho_{k}-\rho_{j}\right) .
$$

LEMma 3.2. Assume that the rational function $R(w)$ has only first order poles and let $x$ be any complex quantity. Then

$$
\sum_{j=0}^{q} \frac{D_{j} \Gamma\left(x-\alpha \rho_{j}+n\right)}{\Gamma\left(x-\alpha \rho_{j}\right)}= \begin{cases}0 ; & n=0,1, \cdots, \alpha-2 \\ \alpha^{\alpha-1} ; & n=\alpha-1, \\ \alpha^{\alpha}(x-\beta) ; & n=\alpha\end{cases}
$$

where $\alpha$ and $\beta$ are defined_by (2.2). 
PROOF. If we compare the coefficients in the Laurent expansion of $R(w)$ obtained from (3.2) with those obtained from (3.1) we have the identities

$$
\sum_{i=0}^{q}\left(-\rho_{i}\right)^{n} D_{i}= \begin{cases}0 ; & n=0,1, \cdots, \alpha-2, \\ 1 ; & n=\alpha-1, \\ 1 / 2(1-\alpha)-\beta ; & n=\alpha .\end{cases}
$$

Let the quantities $b_{k, n}$ be defined by the identity

$$
\frac{\Gamma(x-\alpha \rho+k)}{\Gamma(x-\alpha \rho)}=\sum_{n=0}^{k} b_{k, n}(-\rho)^{n},
$$

then

$$
b_{k, k}=\alpha^{k} ; \quad b_{k, k-1}=\alpha^{k-1}[k x+1 / 2 k(k-1)],
$$

and

$$
\sum_{j=0}^{q} \frac{D_{j} \Gamma\left(x-\alpha \rho_{j}+k\right)}{\Gamma\left(x-\alpha \rho_{j}\right)}=\sum_{j=0}^{q} D_{i} \sum_{n=0}^{k} b_{k, n}\left(-\rho_{j}\right)^{n} .
$$

By interchanging the order of summation in (3.7) and using (3.5) and (3.6), the assertion easily follows.

4. The recursion formula. The reader will readily note that by applying the recurrence relation $\Gamma(x+1)=x \Gamma(x)$ to (1.1) we immediately obtain the difference equation

$$
{ }_{p} g_{q}(w+1)=R(w)_{p} g_{q}(w) .
$$

This is the difference equation referred to in $\$ 1$. We now use (4.1) to prove the following

TheOREM 4.1. Let $c_{m}(m=0,1,2, \cdots)$ be the constants in the factorial expansion of ${ }_{p} g_{q}(w)$ as defined by (2.1). Provided $\rho_{j} \neq \rho_{k}$ for $j \neq k$ we have the recursion formula

$$
c_{m}=\frac{-1}{m \alpha^{\alpha}} \sum_{n=0}^{m-1} c_{n} e(m, n),
$$

where

$$
e(m, n)=\sum_{j=0}^{q} \frac{D_{j} \Gamma\left(\beta-\alpha \rho_{j}+\alpha+m\right)}{\Gamma\left(\beta-\alpha \rho_{j}+n\right)} .
$$

Here $c_{0}=1, \alpha$ and $\beta$ are defined in (2.2), and $D_{j}$ is defined by (3.3).

Proof. We apply Theorem 2.1 to the two sides of (4.1) and obtain 
two different expansions for ${ }_{p} g_{q}(w+1)$ :

$$
\begin{aligned}
& { }_{p} g_{q}(w+1) \\
& =C \alpha^{\alpha w+\alpha}\left[\sum_{m=0}^{M} \frac{c_{m}}{\Gamma(\alpha w+\beta+\alpha+m)}+O\left(\frac{1}{\Gamma(\alpha w+\beta+\alpha+M+1)}\right)\right], \\
& \quad=C \alpha^{\alpha w} R(w)\left[\sum_{m=0}^{M} \frac{c_{m}}{\Gamma(\alpha w+\beta+m)}+O\left(\frac{1}{\Gamma(\alpha w+\beta+M+1)}\right)\right],
\end{aligned}
$$

where $R(w)$ is defined in (3.1) and $C=\left((2 \pi)^{1 / 2}\right)^{1-\alpha} \alpha^{\beta-1 / 2}$. We may rewrite (4.5) in the form:

$$
\begin{aligned}
{ }_{p} g_{q}(w+1)=C \alpha^{\alpha w}\left[\sum_{j=0}^{q} D_{j} \sum_{m=0}^{M} \frac{c_{m}}{\left(w+\rho_{j}\right) \Gamma(\alpha w+\beta+m)}\right. \\
\left.\quad+O\left(\frac{1}{\Gamma(\alpha w+\beta+\alpha+M+1)}\right)\right] .
\end{aligned}
$$

Let $\rho=\min _{j} \operatorname{Re}\left(\rho_{j}\right)$ and by $H$ denote the half-plane consisting of points $w$ such that $\operatorname{Re}(w+\rho)>0$. It then follows from Lemma 3.1 that

$$
\begin{aligned}
{ }_{p} g_{q}(w & +1)=C \alpha^{\alpha w+1}\left[\sum_{m=0}^{M} c_{m} \sum_{n=0}^{\infty} \frac{1}{\Gamma(\alpha w+\beta+m+n+1)}\right. \\
& \left.\times \sum_{j=0}^{q} D_{j} \frac{\Gamma\left(\beta+m-\alpha \rho_{j}+n\right)}{\Gamma\left(\beta+m-\alpha \rho_{j}\right)}+O\left(\frac{1}{\Gamma(\alpha w+\beta+\alpha+M+1)}\right)\right],
\end{aligned}
$$

provided $w$ is in $H$. The summation over $n$ may be restricted to $n \geqq \alpha-1$ according to Lemma 3.2. We set $k=n-\alpha+1$ and obtain

$$
\begin{aligned}
p g_{q}(w+1)=C \alpha^{\alpha w+1}\left[\sum_{m=0}^{M} c_{m} \sum_{k=0}^{\infty} \frac{e(m+k-1, m)}{\Gamma(\alpha w+\beta+\alpha+k+m)}\right. \\
\left.+O\left(\frac{1}{\Gamma(\alpha w+\beta+\alpha+M+1)}\right)\right] .
\end{aligned}
$$

Here the summation over $k$ may be restricted to $k \leqq M-m$ because the remainder can be absorbed in the order term. By introducing $n=m+k$ and interchanging the order of summation we obtain

$$
\begin{aligned}
& p g_{q}(w+1)=C \alpha^{\alpha w+1}\left[\sum_{n=0}^{M} \frac{1}{\Gamma(\alpha w+\beta+\alpha+n)}\right. \\
&\left.\times \sum_{m=0}^{n} c_{m} e(n-1, m)+O\left(\frac{1}{\Gamma(\alpha w+\beta+\alpha+M+1)}\right)\right],
\end{aligned}
$$


provided $w$ is in $H$. We interchange $m$ and $n$ in (4.6) and then compare coefficients with those of (4.4). We have

$$
\alpha^{\alpha-1} c_{m}=\sum_{n=0}^{m} c_{n} e(m-1, n) .
$$

Using (3.4) we can write the last two terms of (4.7) separately and after cancelling the terms in $c_{m}$ and replacing $m-1$ by $m$ the result (4.2) follows.

5. Generalization. The assumption that $\rho_{j} \neq \rho_{k}$ for $j \neq k$ involves no real loss of generality. J. H. B. Kemperman [3] has shown that $c_{m}$ is a polynomial in the parameters $\rho_{j}$. It can be verified that $e(m, n)$ is also a polynomial in the $\rho_{j}$. Hence, if we let $e^{*}(m, n)$ and $c_{m}^{*}$ denote the limiting values as two or more $\rho$ values tend to equality, we can write

$$
c_{m}^{*}=\frac{-1}{m \alpha} \sum_{n=0}^{m-1} c_{n}^{*} e^{*}(m, n) .
$$

The calculation of $e^{*}(m, n)$ by the limiting procedure is difficult. The recurrence relation for the case that two or more values of $\rho$ are equal may also be obtained by using the factorial representations of nonlinear terms of the partial fraction expansion of $R(w)$. In fact, this direct approach is much easier.

\section{REFERENCES}

1. W. B. Ford, The asymptotic developments of functions defined by Maclaurin series, University of Michigan Studies, Scientific Series, vol. 11, 1936.

2. H. K. Hughes, The asymptotic developments of a class of entire functions, Bull. Amer. Math. Soc. vol. 51 (1945) pp. 456-461.

3. J. H. B. Kemperman, Unpublished notes from a seminar first presented at Purdue University in 1952.

4. H. Van Engen, Concerning gamma function expansions, Tôhoku Math. J. vol. 45 (1939) pp. 124-129.

5. E. M. Wright, The asymptotic expansion of the generalized hypergeometric function, Proc. London Math. Soc. (2) vol. 46 (1940) pp. 389-408.

Purdue University 